\title{
Amorphous Silicon Pixel Radiation Detectors And Associated Thin Film Transistor Electronics Readout
}

V. Perez-Mendez, J. Drewery, W.S. Hong. T. Jing, S.N. Kaplan, H. Lee, A. Mireshghi

Physics Division

\author{
Lawrence Berkeley Laboratory \\ University of California \\ Berkeley, CA 94720
}

October 1994

\section{MASTER}

This work was supported by the Director, Office of Energy Research, Office of High Energy and Nuclear Physics, and Office of Health and Environmental Research, Division of Physics and Technological Research of the US. Department of Energy under contract No. DE-AC03-76SF00098.

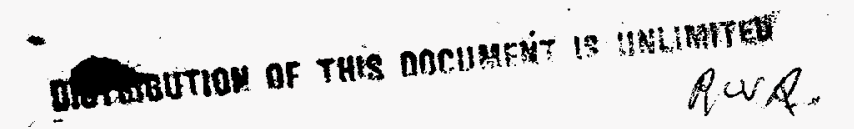




\section{DISCLAIMER}

This report was prepared as an account of work sponsored by an agency of the United States Government. Neither the United States Government nor any agency thereof, nor any of their employees, make any warranty, express or implied, or assumes any legal liability or responsibility for the accuracy, completeness, or usefulness of any information, apparatus, product, or process disclosed, or represents that its use would not infringe privately owned rights. Reference herein to any specific commercial product, process, or service by trade name, trademark, manufacturer, or otherwise does not necessarily constitute or imply its endorsement, recommendation, or favoring by the United States Government or any agency thereof. The views and opinions of authors expressed herein do not necessarily state or reflect those of the United States Government or any agency thereof. 


\title{
DISCLAIMER
}

\begin{abstract}
Portions of this document may be illegible in electronic image products. Images are produced from the best available original document.
\end{abstract}




\title{
Amorphous Silicon Pixel Radiation Detectors And Associated Thin Film Transistor Electronics Readout
}

\author{
V. Perez-Mendez, J. Drewery, W.S. Hong. T. Jing, S.N. Kaplan, H. Lee, A. Mireshghi \\ Lawrence Berkeley Lab, University of California, Berkeley, CA 94720
}

\begin{abstract}
We describe the characteristics of thin $(1 \mu \mathrm{m})$ and thick $(>30 \mu \mathrm{m})$ hydrogenated amorphous silicon $p$-i-n diodes which are optimized for detecting and recording the spatial distribution of charged particles, $x-$ rays and $\gamma$ rays. For $x$-ray, $\gamma$ ray, and charged particle detection we can use thin $p-i-n$ photosensitive diode arrays coupled to evaporated layers of suitable scintillators. For direct detection of charged particles with high resistance to radiation damage, we use the thick $p-i-n$ diode arrays. Deposition techniques using helium dilution, which produce samples with low stress are described. Pixel arrays for flux exposures can be readout by transistor, single diode or two diode switches. Polysilicon charge sensitive pixel amplifiers for single event detection are described. Various applications in nuclear, particle physics, $x$-ray medical imaging, neutron crystallography, and radionuclide chromatography are discussed.
\end{abstract}




\section{INTRODUCTION}

Thin layers of hydrogenated amorphous silicon (a-Si:H) with thickness $0.5-1$ $\mu \mathrm{m}$ have found extensive application in solar cells and in thin film transistors (TFT). A well known application of thick $>30 \mu \mathrm{m}$ layers of a-Si:H is to electrophotography devices. In all these devices the usual configuration is that of a $p-i-n$ diode with very thin $(50 \mathrm{~nm}) \mathrm{p}^{+}$and $\mathrm{n}^{+}$doped layers and the bulk consisting of intrinsic a-Si:H. As shown in Fig. 1, for radiation detection we use the same general configuration of a reverse biased p-i-n diode. In many of the applications that are used in physics, medical imaging and biology, the spatial distribution of the incident radiation is important: hence we use pixel or strip configurations with appropriately shaped metallic contacts. In some applications single particles are detected; the detector array then requires individual, low noise polysilicon TFT amplifiers attached to each pixel. Other applications are to radiation flux detection: for these, simple routing electronics may be sufficient. Charged particle detection - specifically minimum ionizing particles (MIPs) can be accomplished by use of $p-i-n$ diodes with thick $i$ layers in which the charged particle can produce a sufficient number of electron-hole pairs by direct interaction in the depleted $\mathrm{i}$ layer. An alternative scheme for MIPs detection is to use pixel/strip arrays of thin a-Si:H diode layers with transparent metallic or tin oxide contacts which function as visible photon sensors. These are coupled to layers of light emitting (scintillator) material, such as cesium iodide. For the detection of $\mathrm{x}$-rays or $\gamma$ rays of energy above a few $\mathrm{KeV}$, the scintillator - a-Si:H combination is favored due to the low interaction probability of the radiation with a low $z$ element such as silicon. For detection of $x$-rays in $x$-ray crystallography applications $(\mathrm{E} \gamma=8 \mathrm{KeV}$ ), it is also possible to use moderately thick layers of a$\mathrm{Si}: \mathrm{H} 90 \%$ a-Ge:H (20\%) where the germanium is the high $\mathrm{z}$ element contributing to the interaction. 


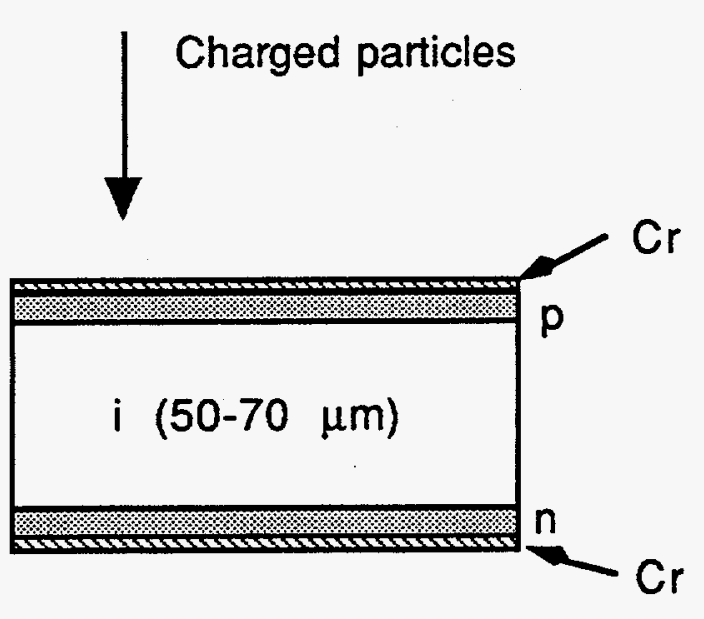

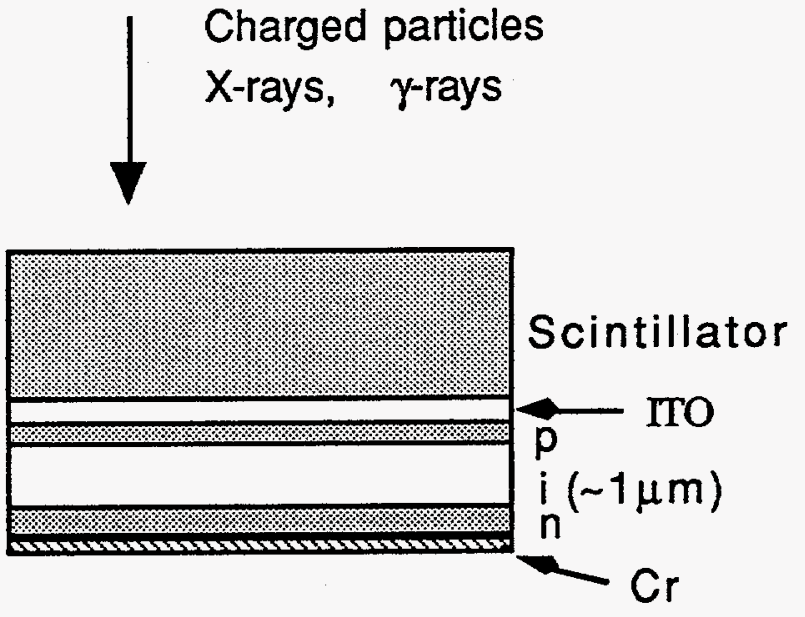
$\mathrm{X}$-rays, $\boldsymbol{\gamma}$-rays

Fig. 1. Detector configurations (a) thick $p-i-n$ diode (b) thin $p-i-n$ photodiode coupled to scintillator layer.

The potential usefulness of amorphous silicon as a detector material is due to the following (a) It is readily made by inexpensive plasma enhanced chemical vapor deposition (PECVD) techniques in large areas $>30 \times 30 \mathrm{~cm}$ : (b) it is very radiation resistant to fast neutrons, charged particles and $\gamma$ rays: (c) dispersed thin film transistor (TFT) electronics for reading out pixel or strip arrays is readily made by the same PECVD technologies in an integral manner.

\section{Deposition techniques for a-Si:H p-i-n detector diodes}

The properties of the detector diodes are determined to a large extent by their structure and their electrical and mechanical properties which are dependent on the deposition technique.

All of our detectors are operated as reverse biased $p-i-n$ diodes, made in a PECVD machine operating at $85 \mathrm{MHz}$. The $\mathrm{n}+$ layer $\sim 50 \mathrm{~nm}$ thick is deposited on a chromium or tin oxide semi transparent contact on 7059 glass or on Kapton. The $\mathrm{i}$ layer is $\sim 1-5 \mu \mathrm{m}$ thick for photodiodes and 30-50 $\mu \mathrm{m}$ thick for charged particle detection. A p+layer $\sim 25 \mathrm{~nm}$ thick is used for photodiode applications in 
order not to absorb too much light. A p+layer $\sim 300-400 \mathrm{~nm}$ thick is used for the 30-50 $\mu \mathrm{m}$ thick diodes to produce a greater space between the metal and the $p-i$ interface where the $E$ field is largest. A chromium or tin oxide transparent layer is deposited on top. As discussed in the following section a density of dangling bonds $2 \times 10^{15}$ is required for full depletion of layers $>30 \mu \mathrm{m}$ thick. Table 1 below gives the deposition conditions of the $\mathrm{i}$ layer. Using pure silane as the deposition gas for the $i$ layer is satisfactory for the photodiode ( $i$ layer $\sim 1-5 \mu \mathrm{m}$ ) case. For making thick layers it is necessary to reduce the stress in the deposited layer to prevent peeling off. The material as deposited under the conditions of Table I has the required lower stress but a higher than acceptable density of dangling bond states. These can be reduced to $N_{d}{ }^{*}=5 \times 10^{14} / \mathrm{cm}^{3}$ by annealing at $160^{\circ} \mathrm{C}$ in air for 100 hours(1); the stress does not change in the annealing process.

An added advantage of the Helium dilution deposited material is that the deposition rate is $\sim 3-4 \mu \mathrm{m} / \mathrm{hour}$.(2)

Table I

\begin{tabular}{|c|c|c|c|c|c|}
\hline Gas & Temperature & Power dens & Fyleposition I & qutress & $\begin{array}{l}\mathrm{Nd}^{*} \\
\left(\mathrm{~cm}^{-3}\right)\end{array}$ \\
\hline $\mathrm{SiH} 4(100 \%)$ & $250^{\circ}$ & $45 \mathrm{~mW} / \mathrm{cm}^{2}$ & $2 \mu \mathrm{m} /$ hour & $360 \mathrm{Mpa}$ & $5 \times 10^{14}$ \\
\hline $\begin{array}{l}\operatorname{SiH} 4(.60 \%) \\
\mathrm{He}(40 \%)\end{array}$ & $150^{\circ}$ & $95 \mathrm{~mW} / \mathrm{cm}^{2}$ & $4 \mu \mathrm{m} /$ hour & $110 \mathrm{Mpa}$ & $3 \times 10^{15}$ \\
\hline
\end{tabular}

\section{DETECTION OF CHARGED PARTICLES WITH THICK P-I-N DIODES}

A reverse biased diode with a thick i layer requires use of a-Si:H with a low density of dangling bonds $\left(<2 \times 10^{15} / \mathrm{cm}^{3}\right)$ for the following reasons: (a) The mean free path of electrons and holes is $d=\mu \tau E$ where $\mu, \tau$, are the mobilities and lifetimes of the electrons or holes and $E=$ the electric field of the external bias; therefore a large value of $\mu \tau$, is desirable since $\mu \tau \mathrm{N}_{d} \approx 2.5 \times 10^{8}$ in i layers made using pure silane gas(3). (b) When an external bias is applied, a fraction of the 
neutral dangling bonds $\mathrm{Nd}^{*} \approx 0.3 \mathrm{Nd}$. (4) are ionized and a residual positive charge remains. This fixed charge causes the electric field in the $i$ layer to drop linearly with distance, until a low electric field $<5 \times 10^{4} \mathrm{v} / \mathrm{cm}$ is reached at which point the $E$ field decreases exponentially (5). Hence, in order to have a fully depleted detector, a minimum bias is needed which increases proportionately to the density of dangling bonds. The electric field peaks at the $p-i$ interface which is close to the metal contact and at high biases enhances breakdown. The following schemes to promote full depletion without breakdown have been developed $(5,6)$ as shown in Fig 2. (a) The p layer is made considerably thicker, i.e., 200-400 $\mathrm{nm}$ which places the peak $E$ field further away from the metal contact. (b) A buried, thin $\sim 20 \mathrm{~nm}$ thick $p$ layer is deposited with a $\sim 1 \mu \mathrm{m}$ spacing to the electrode. Schemes (a) and (b) increase the allowable bias potential by $\sim 3$ times. (c) One or more thin $p$ layers are deposited in the middle of the $i$ layer whose effect is to decrease the effective slope of the electric field since the $p$ layer, under bias, leaves a residual negative charge. With one $p$ central layer of the appropriate thickness, the applied bias for full depletion can be lowered by a factor of 2, (7). Schemes (a) and (b) cause little or no loss in the number of charged carriers traversing the i layer. Scheme (c) causes some electron loss due to trapping because of the lower mean free path in the $p$ layer whereas the hole loss is minimal (8).

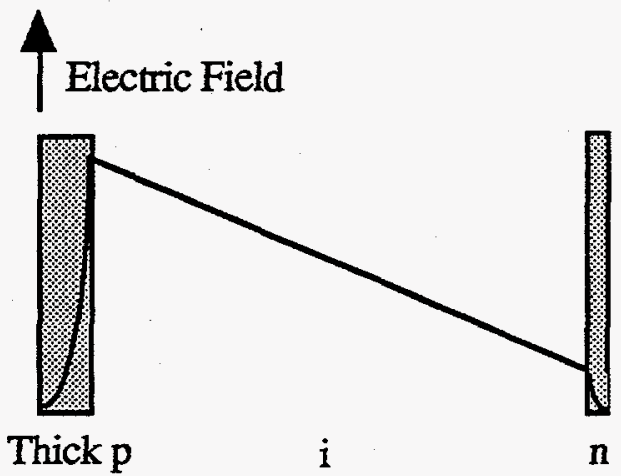

(a)

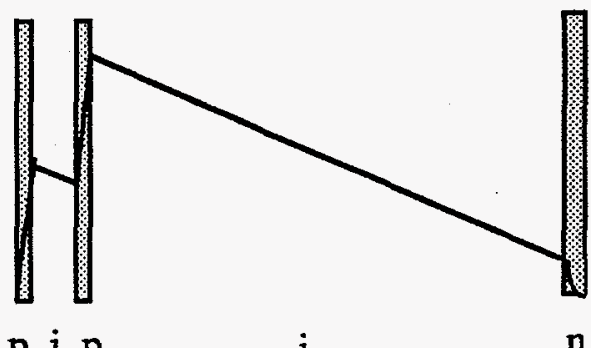

(b) 


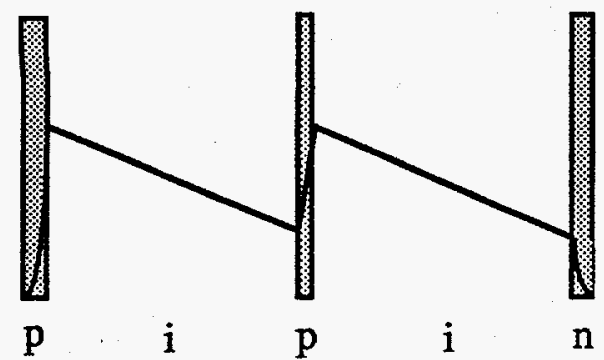

(c)

Fig. 2. Full depletion schemes (a) thick $p$ layer (b) buried $p$ layer (c) central $p$ layer.

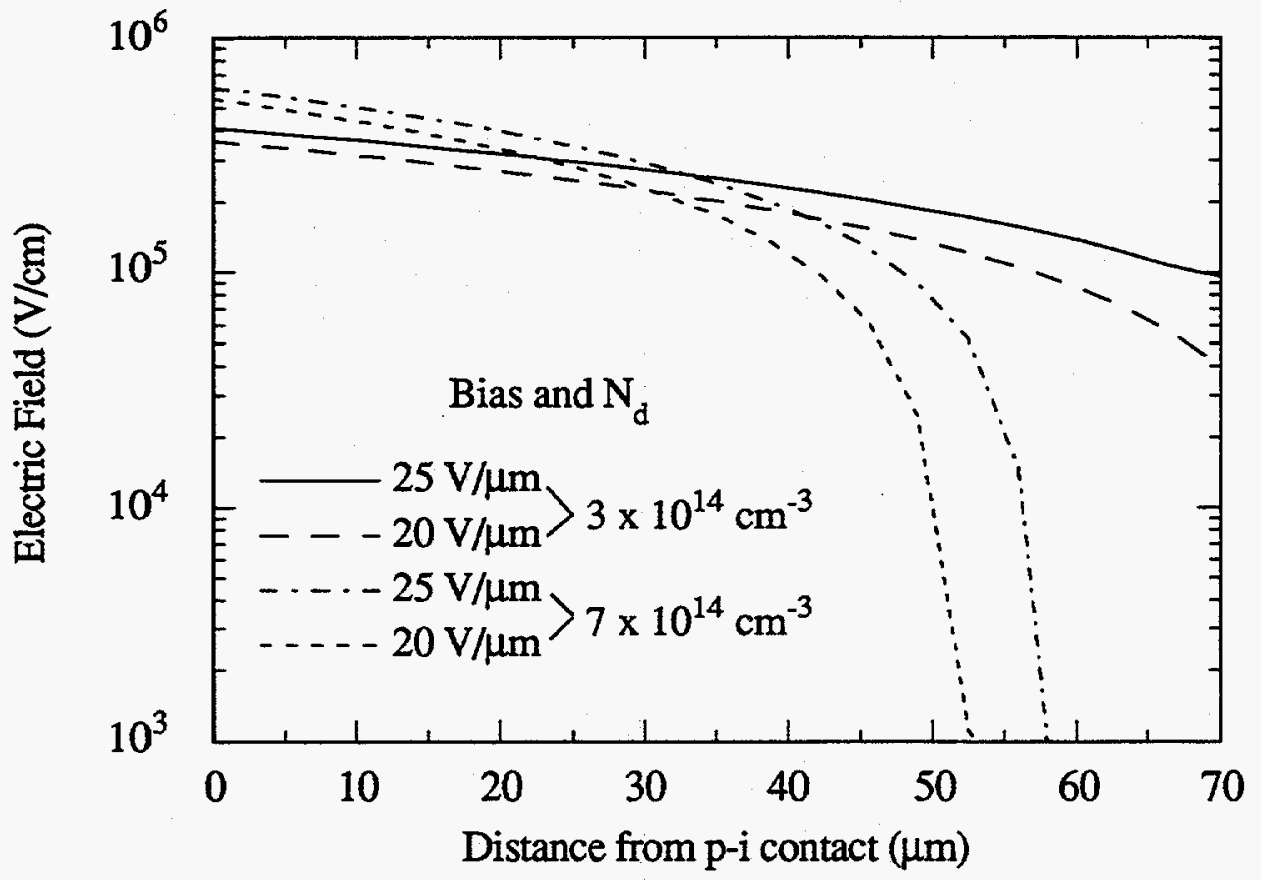

Fig. 3. Electric field in i-layer of a-Si:H pin diode calculated for two different bias and densities of ionized gangling bonds.

Fig. 3 shows the depletion widths achieved with different values of ionized dangling bonds $\mathrm{N}_{d}{ }^{*}$ and bias voltages. Another difficulty in making thick $p-i-n$ layers was that in the conventional deposition technique, the a-Si:H was produced with a high stress which tended to cause it to peel off the substrate. The helium diluted deposition technique described in the previous section solves this problem. 
An important quantity in evaluating the interaction of charged particles with an aSi:H $\mathrm{p}-\mathrm{i}-\mathrm{n}$ diode is $\mathrm{W}=$ average energy used in producing $1 \mathrm{e}, \mathrm{h}$ pair. We measured $\mathrm{W}$ using an $860 \mathrm{MeV}$ alpha particle beam - essentially MIPs (10) and found W = 4.8 0.3 $\mathrm{eV}$. This corresponds to a production rate by MIPs of $\sim 80 \mathrm{e}, \mathrm{h}$ pairs $/ \mu \mathrm{m}$ of a-Si:H.

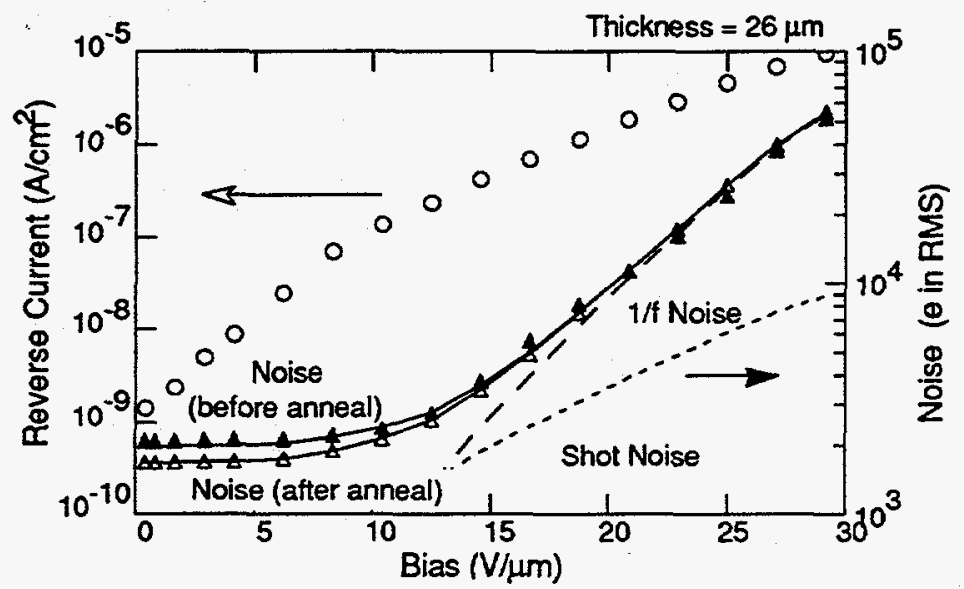

(a)

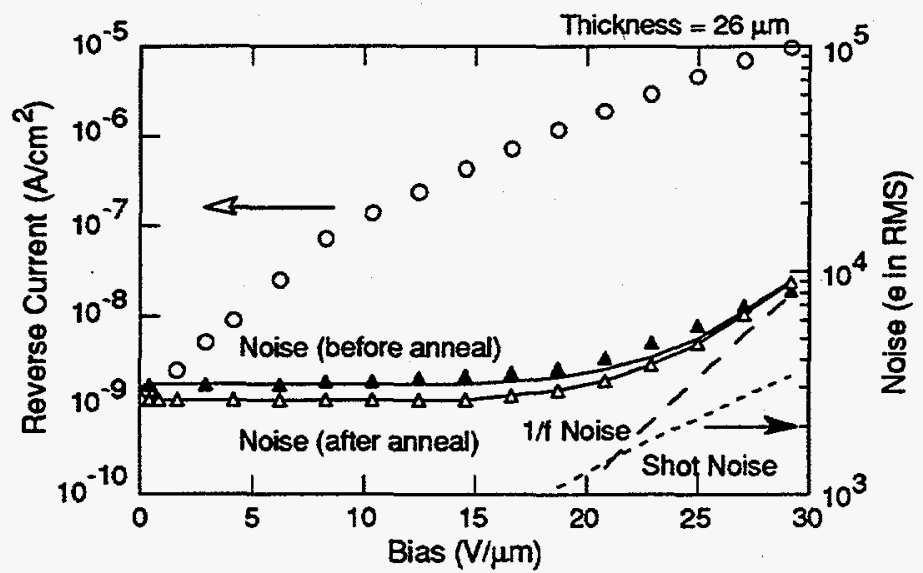

(b)

Fig. 4. Resistive, shot and $1 / \mathrm{f}$ noise in a-Si:H p-i-n detector diode before and after annealing at a shaping time of $2.5 \mu \mathrm{sec}$.

The noise produced in a reverse biased $\mathrm{p}-\mathrm{i}-\mathrm{n}$ detector together with that of a typical readout amplifier should be small. In Fig. 4 we show the noise in a $26 \mu \mathrm{m}$ diode as a function of reverse bias, measured by a charge sensitive amplifier with a $2.5 \mu \mathrm{sec} C R-$ $(\mathrm{RC})^{2}$ shaping time. The flat portion of the noise graph, at low biases, is the sum of (a) the amplifier noise when loaded by the capacity of the detector, and a mostly resistive 
(Nyquist) noise generated by the contact resistance, and the $p$ layer resistance. The noise contribution of the $p$ layer resistance can be reduced by annealing ( 2 hours at $180^{\circ} \mathrm{C}$ ) under bias (9) At higher biases when the reverse current increases, the contributing shot noise - which has a flat frequency spectrum and is proportional to the current is observed. At still higher biases with larger reverse current, 1/f noise, which has the $1 / f$ spectral response and is proportional to $R^{2}$ becomes the predominant contribution. All of these noise components are proportional to the area of a pixel detector (10).
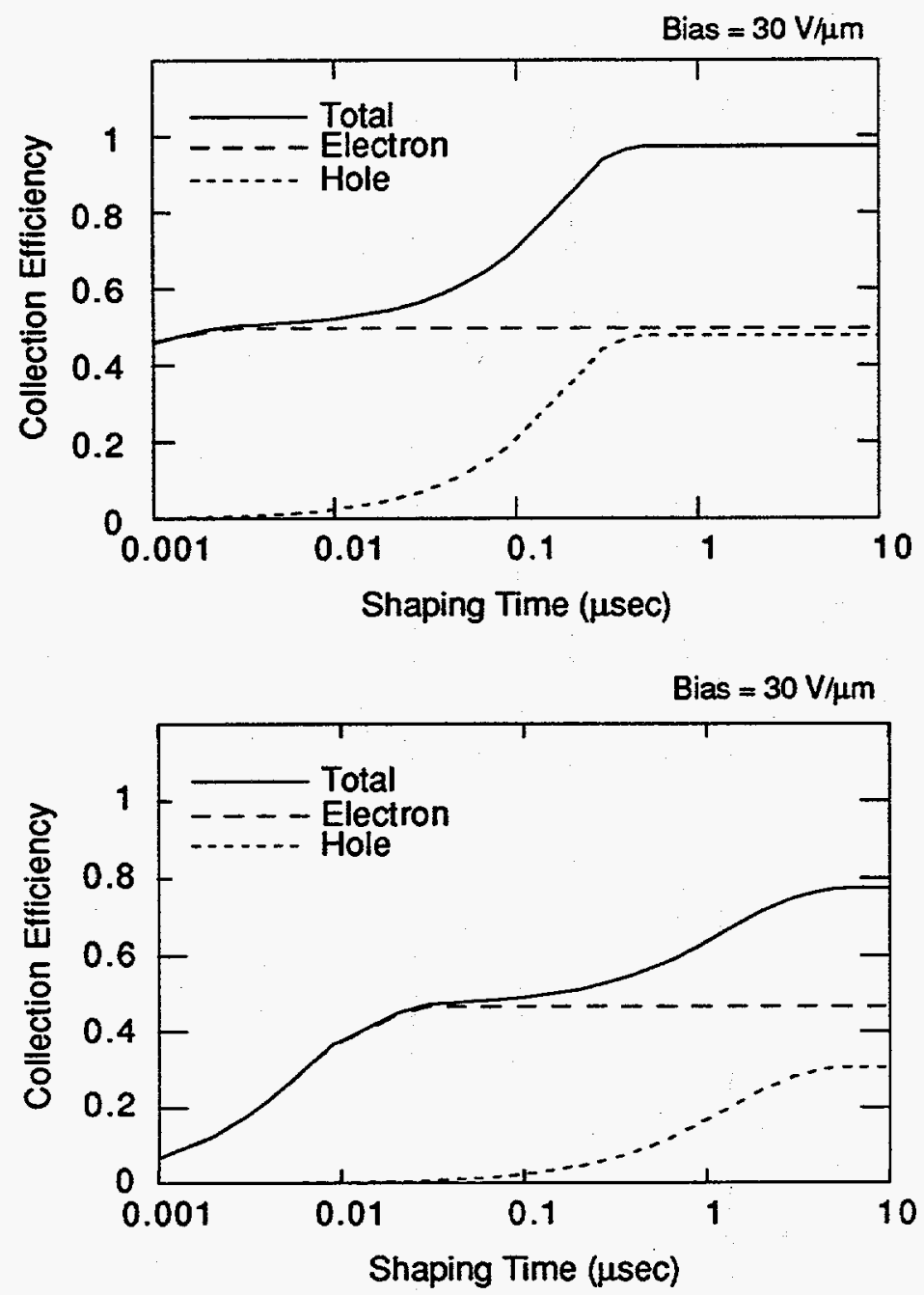

Fig. 5. Electron and hole collection time in $1 \mu \mathrm{m}$ and $50 \mu \mathrm{m}$ thick detector diode at $30 \mathrm{~V}$ bias. 
The signal response as a function of shaping time can be calculated from the mobility - typically $\mu_{\mathrm{e}} \sim 1 \mathrm{~cm}^{2} \mathrm{Nsec}, \mu_{\mathrm{h}} \sim 0.005 / \mathrm{cm}^{2} / \mathrm{Vsec}$, and the $E$ field in the i layer at a given bias. Fig. 5 shows the signal from electrons and holes for $1 \mu \mathrm{m}$ and for 50 $\mu \mathrm{m}$ thick diodes. For the $50 \mu \mathrm{m}$ thick diode, fast timing is achieved by collecting the electron signal only in $<10$ nsec

\section{DETECTION OF RADIATION BY THIN A-SI:H DIODES COUPLED TO SCINTILLATOR LAYERS}

For this case we assume that the signal is produced predominantly by the interaction of the radiation with the scintillator and that the scintillation light is then detected by the a-Si:H through a transparent indium tin oxide contact. A further requirement - if good position accuracy in a pixel or strip detector is desired - is that the scintillation light be suitably collimated. We have worked primarily with evaporated layers of $\mathrm{C}_{S}$ l activated with thallium. The $\mathrm{C}_{\boldsymbol{S}}(\mathrm{TI})$ has been measured (11) to produce > 50,000 visible light photons $/ \mathrm{MeV}$ of radiation interaction - charged particles, $\mathrm{x}$-rays or $\gamma$ rays. The spectral emission of $C_{S}(T I)$ is in the $400-700 \mathrm{~nm}$ range for which a $1 \mu \mathrm{m}$ thick a-Si:H diode has a flat response. For the $\mathrm{C}_{\mathrm{S}} \mathrm{I}(\mathrm{TI}) / \mathrm{a}-\mathrm{Si}: \mathrm{H}$ combination, the light to e,h pairs in the a-Si:H photodiode is $>70 \%$. Some light collimation was achieved by inducing some structure to develop through the $\mathrm{C}_{\mathbf{S}}(\mathrm{TI})$ layer by controlling the cooling rate of the substrate in the evaporation process $(12,13)$. We have obtained better light collimation - hence better spatial resolution by evaporating $\mathrm{C}_{\mathrm{S}} \mathrm{I}(\mathrm{TI})$ on to an etched patterned substrate of Polyimide (14) deposited on the a-Si:H surface or on a glass/aluminum substrate, as shown in Fig.6. The point spread function produced by an $\mathrm{x}$-ray beam incident through a $25 \mu \mathrm{m}$ aperture and measured by a linear detector array is shown in Fig.7. The better columnar structure produced by the patterned substrate compared to the thermally induced pattern produces point spread functions which are 2-3 narrower than those from the plain substrate. Another quantity of 
interest is the resistance of the device to radiation. As noted previously, the a-Si:H diodes and TFT are very radiation resistant. $C_{S} I(T I)$ crystals are considerably more susceptible to radiation damage. In general it has been shown that the main loss is due to decrease of light transmission through the bulk of a crystal. We confirmed this by measuring the signal decrease for a $1 \mathrm{~cm}^{3} \mathrm{Cs}(\mathrm{TI})$ crystal and an evaporated layer $200 \mu \mathrm{m}$ thick and we show that the thin layer has a radiation resistance $\sim 100$ higher than the crystal (14).

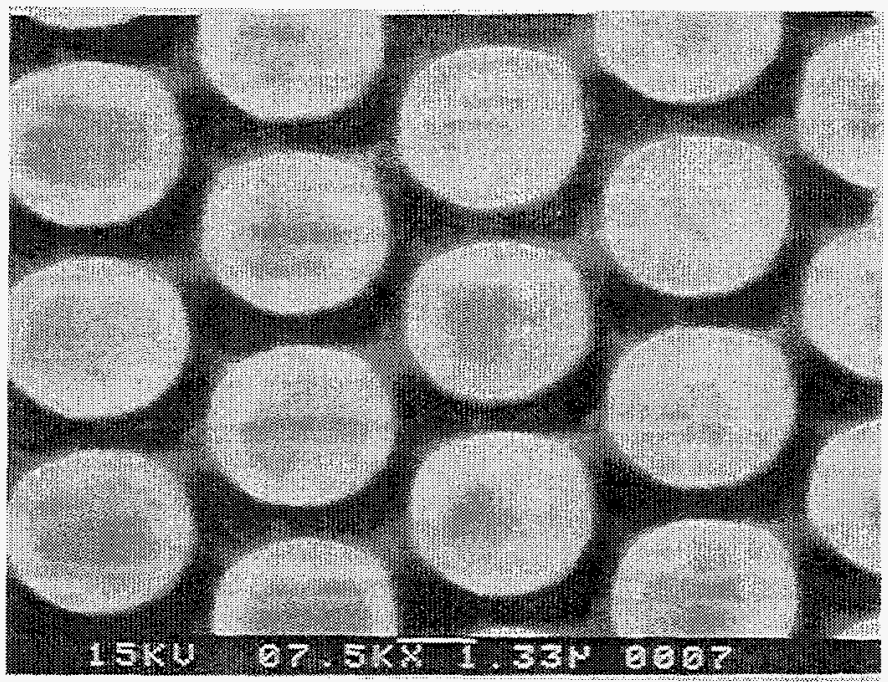

(a)

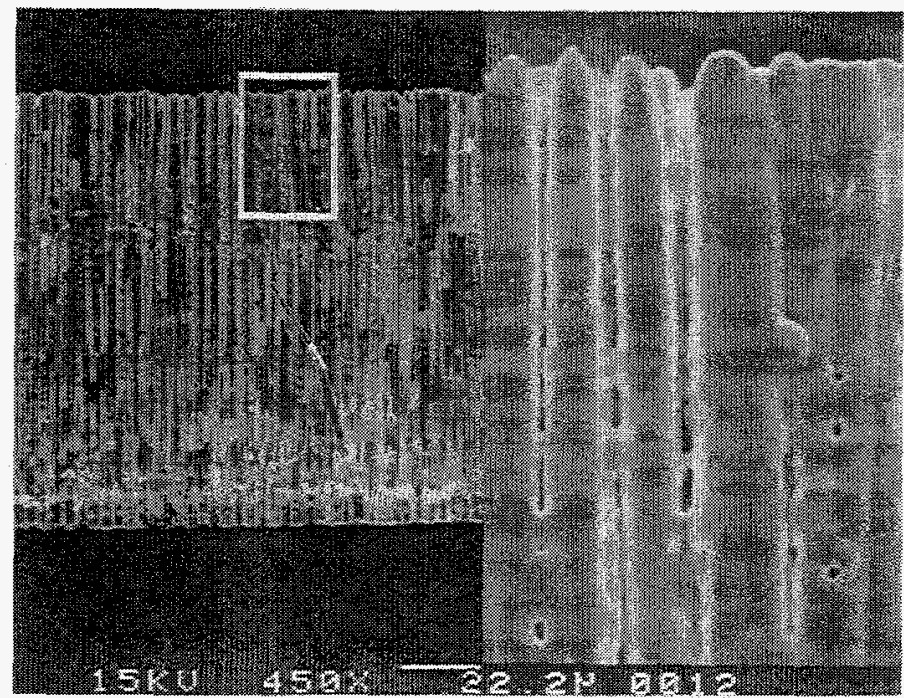

(b)

Fig. 6. Position accuracy in Csl/polyimide grid/a-Si:H combination (a) grid pattern (b) SEM picture of columnar structure in CsI layer. 


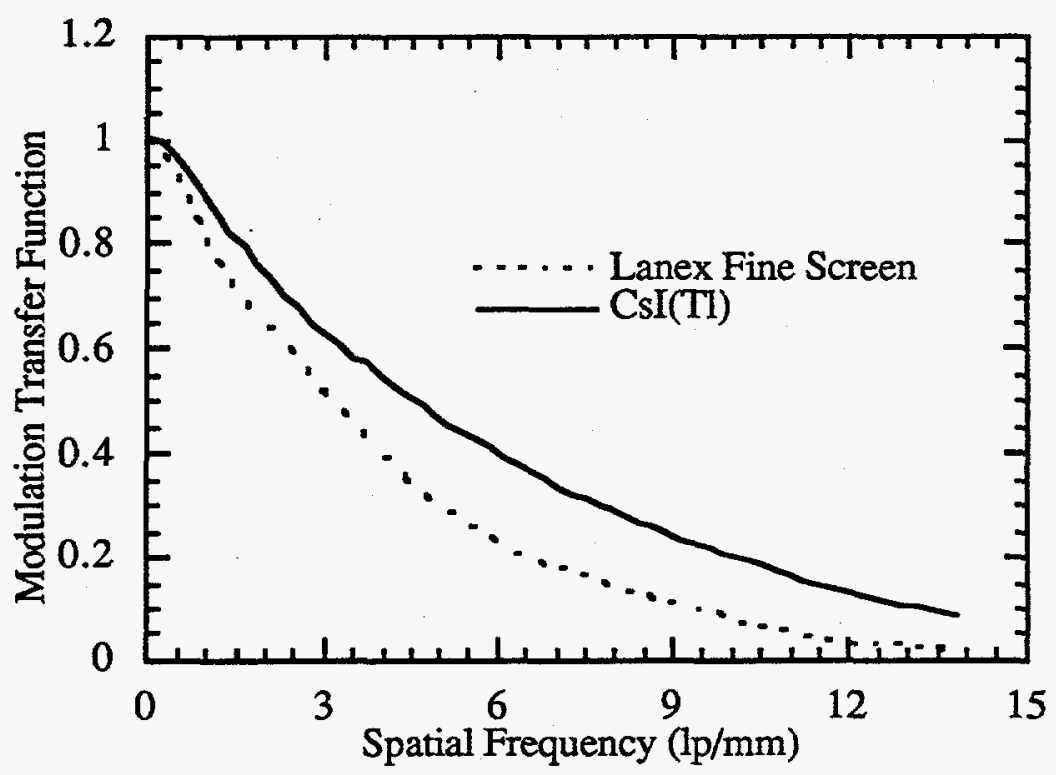

Fig. 7. Point spread function for Csl layers deposited on polyimide dot pattern.

\section{THIN FILM TRANSISTOR (TFT) READOUTS FOR PIXEL ARRAYS}

Fig.(8a) shows a pixel array in which each element is read out by an a-Si:H switching transistor. The array can be read out line by line by sending out an enable signal to the TFT in that line. The charge in the elements in the tagged line can be read out by some integrated circuit chip with multiple inputs such as the SVX chip made at the Lawrence Berkeley Lab. This chip has 128 parallel low noise inputs and other features(15).

Alternative readout methods involve a single diode connected to each element Fig.(8b), or two diodes as shown in Fig.(8c)(16). The two diode readout is faster and has a larger dynamic range than the single diode scheme; it also minimizes switching transients. Diode readouts have the following advantage 
compared to TFT (a) they require less production masks (b) by not needing a $\mathrm{SiO} 2$ or Si3N4 insulator they minimize radiation damage problems.

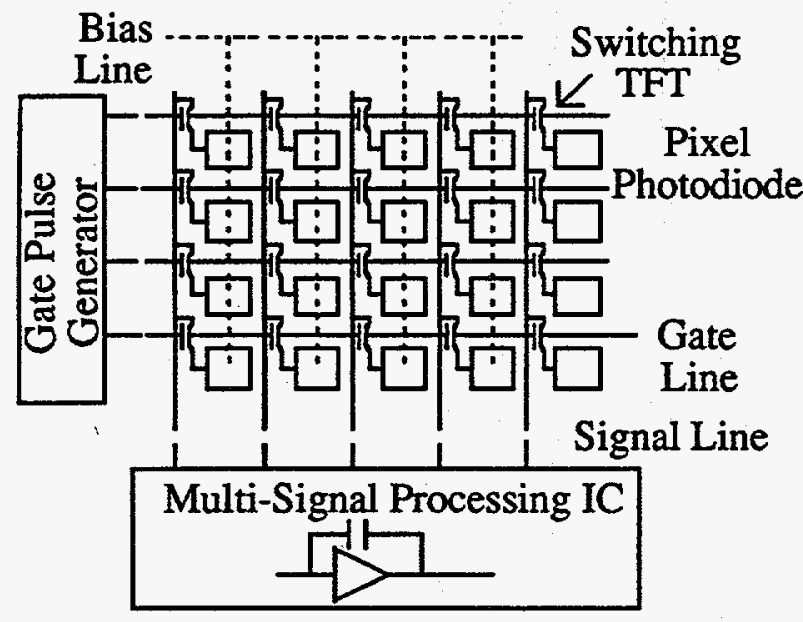

(a)

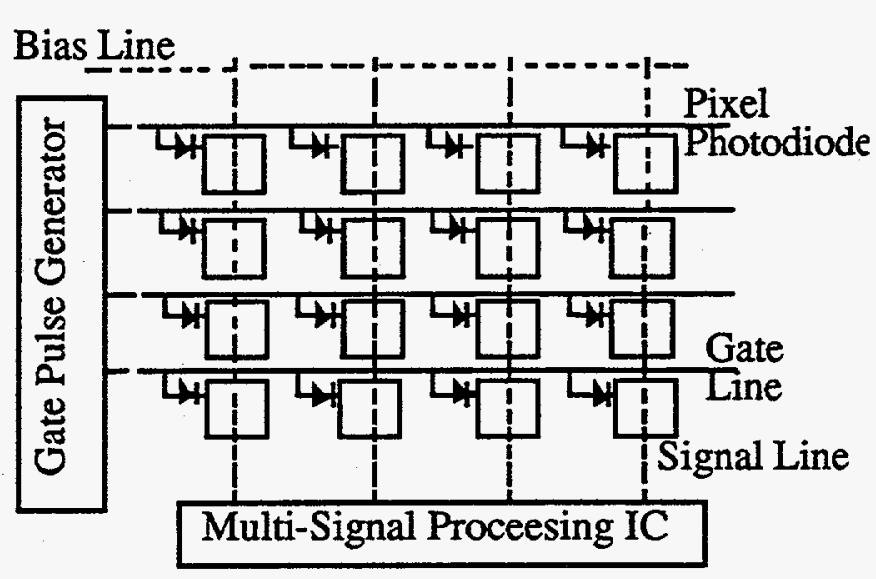

(b)

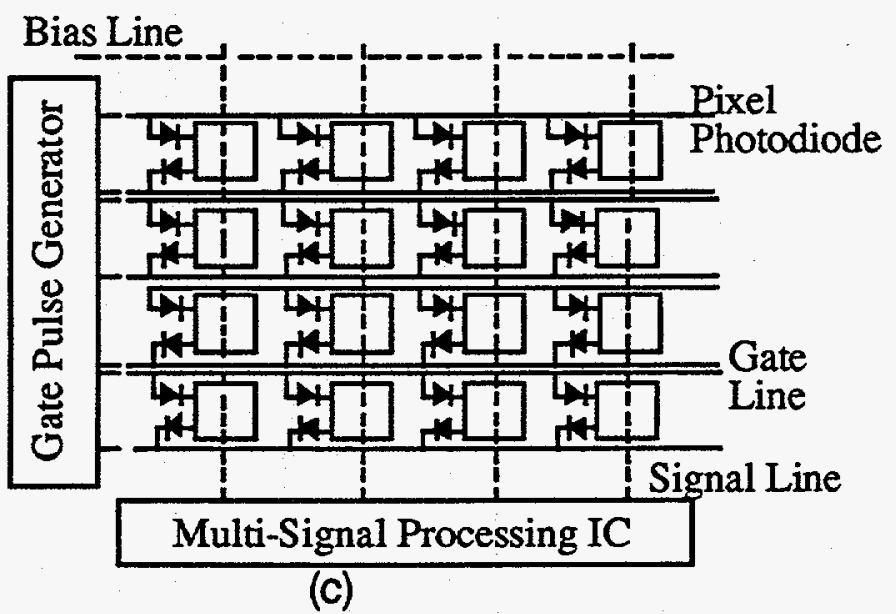

Fig. 8. Diode, TFT readout schemes (a) TFT (b) single diode (c) two diode switchs

As mentioned in the introduction there are some applications requiring the detection of individual charged particles, neutrons or $x$-ray events in which the signal collected in each element needs to be amplified to bring it to a satisfactory level above system noise. This can be accomplished by depositing a TFT 
amplifier made of polysilicon underneath each element of the array, and then reading the output by enabling lines as in the diode case.

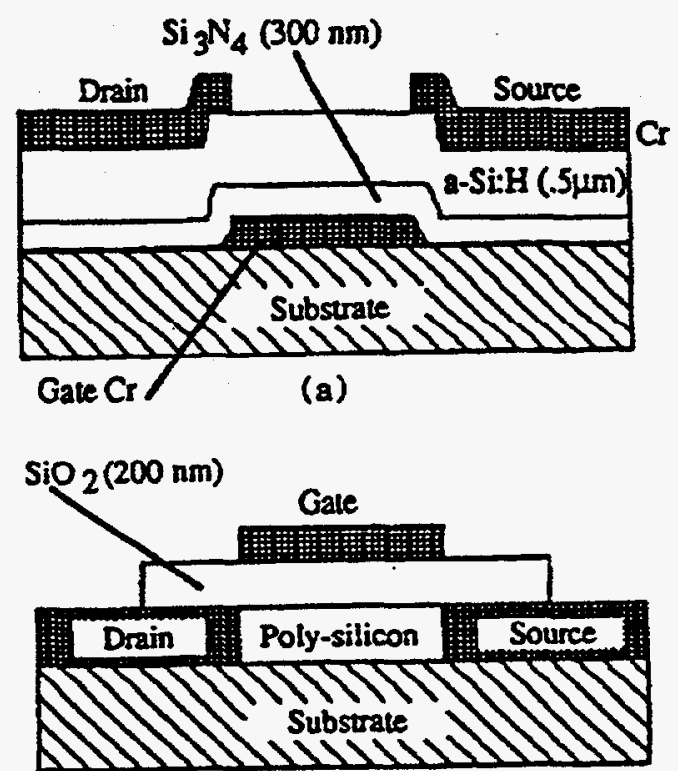

(b)

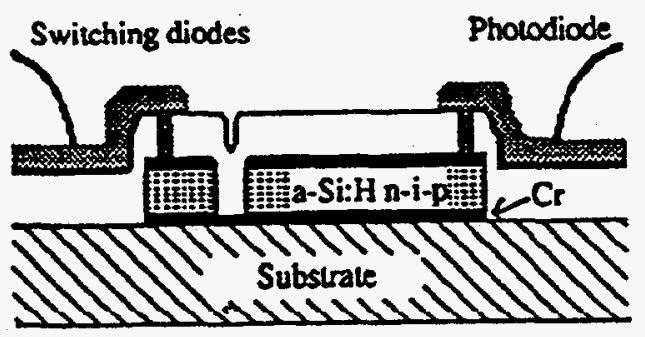

(c)

Fig. 9. Structure of TFTs and diode readout (a) a-Si:H TFT (b) polysilicon TFT (c) diode readout cross section

Amorphous silicon TFT can only be used as switching devices because the threshold voltage of the gate bias shifts continuously under D.C. bias. True CMOS amplifiers can be made using polysilicon TFT since both electron and hole mobilities are comparable and much higher than the corresponding ones for a-Si:H TFT as show in Table II. 
Table II . Typical properties of a-Si:H and polysilicon TFTs

\begin{tabular}{|c|c|c|}
\hline Type & a-Si:H TFT & Poly-Si TFT \\
\hline$\mu \mathrm{e}(\mathrm{cm} 2 \mathrm{Nsec})$ & 1 & 150 \\
\hline$\mu \mathrm{h}(\mathrm{cm} 2 N \mathrm{sec})$ & .005 & 80 \\
\hline $\mathrm{gm}(\mu \mathrm{AN})$ & 5 & 150 \\
\hline Bandwidth (MHz) & 5 & 100 \\
\hline Noise $^{*}(e)$ & 500 & 500 \\
\hline
\end{tabular}

* Equivalent noise charges for an CR-(RC)4 shaping time of $1 \mu \mathrm{sec}$.

Fig.(9) shows the structure of a-Si:H TFT, polysilicon TFT and a cross section of the diode readout. Using the facilities of the Xerox research lab in Palo Alto, we designed and tested 3 stage CMOS polysilicon amplifiers with a charge sensitive input stage followed by a voltage gain stage, followed by a low impedance output stage for driving signals through a pixel array. This prototype amplifier design is shown in Fig.10. The measured characteristics are given below in Table III .(17)

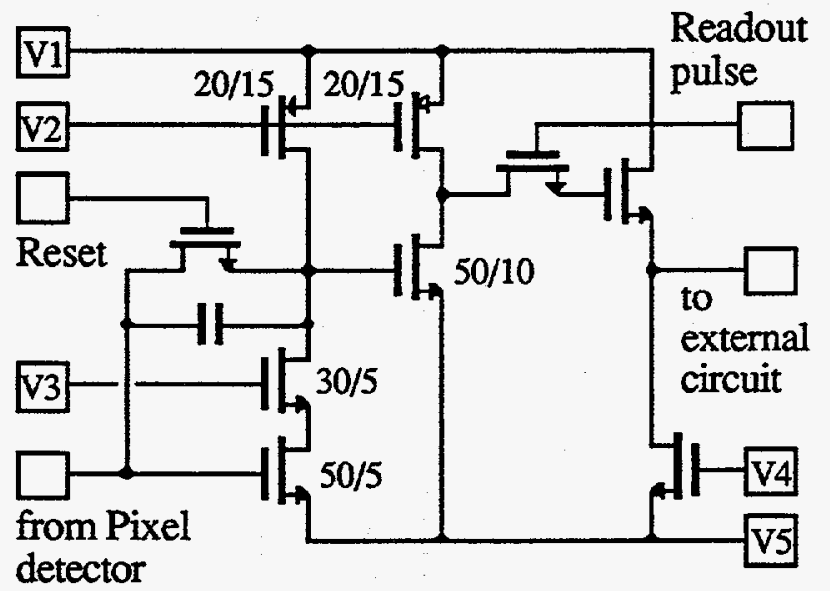

Fig. 10 Polysilicon TFT charge sensitive amplifier 
Table III. Characteristics of polysilicon TFT charge-sensitive amplifier

\begin{tabular}{|l|l|}
\hline \hline Dimension & $<200 \times 200 \mu \mathrm{m} 2$ \\
\hline Charge gain & $\sim 0.02 \mathrm{mV} /$ electron \\
\hline Rise time & $100 \mathrm{nsec}$ \\
\hline Gain-Bandwidth & $350 \mathrm{MHz}$ \\
\hline Noise & $\sim 1000$ electrons \\
\hline Power dissipation & $<1 \mathrm{~mW} /$ pixel \\
\hline
\end{tabular}

\section{DETECTOR APPLICATIONS OF a-Si:H PIXEL ARRAYS}

(a) MIPs Detection in Particle and Nuclear Physics

Particle and nuclear physics experiments require position information using pixel or strip configurations. Most of the time, minimum ionizing particles- MIPS are detected. Two configuration are possible (1) using a detector $>50 \mu \mathrm{m}$ thick in which each MIPs produces $\sim 80$ e,h pairs $/ \mu \mathrm{m}$. As mentioned previously the reduced stress material produced by $\mathrm{He}-\mathrm{SiH} 4$ gas mixtures is well suited for this purpose. (2) The second possibility is to use the scintillator/a-Si:H thin photodiode structure. The scintillator of choice is CsI(TI) with the columnar structure discussed in section 4. A MIP traversing a CsI(TI) layer $1.1 \mathrm{~mm}$ thick (density $4.5 \mathrm{~g} / \mathrm{cm}^{3}$ ) deposits $1 \mathrm{MeV}$ energy within it.

Fig.(11) shows the pulse height distribution produced by the most energetic electrons from Sr-90 source in an a-Si:H diode with ITO top contact and light detection efficiency $\sim 70 \%$. From the mean number of e,h pairs $\sim 20,000$, we deduce that $\sim 40,000$ visible light photons were collected using a correction (18) to convert the CsI(TI) light spectrum distribution to the full range of visible light. 


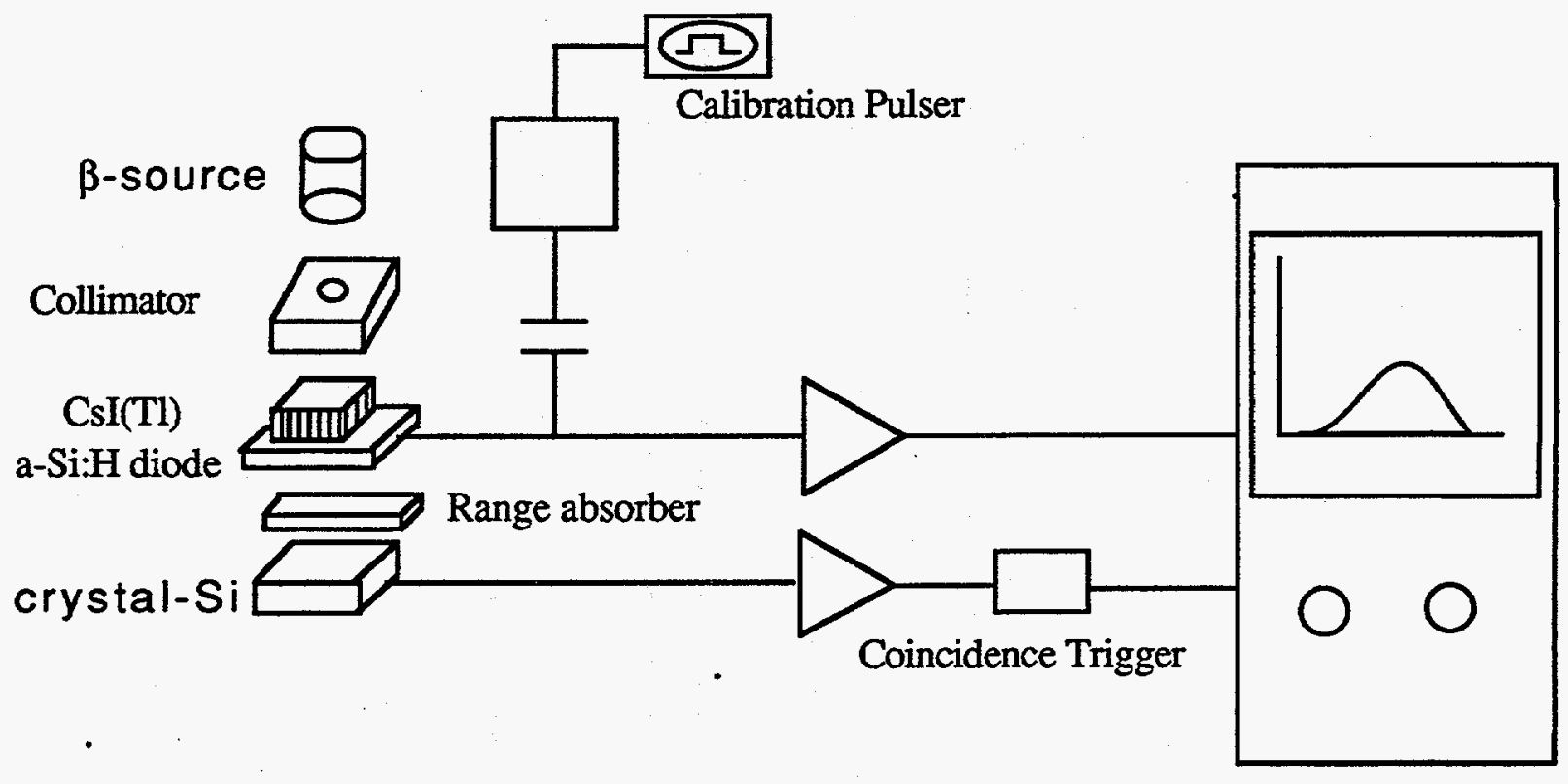

Pulse-Height-Analyser

(a)

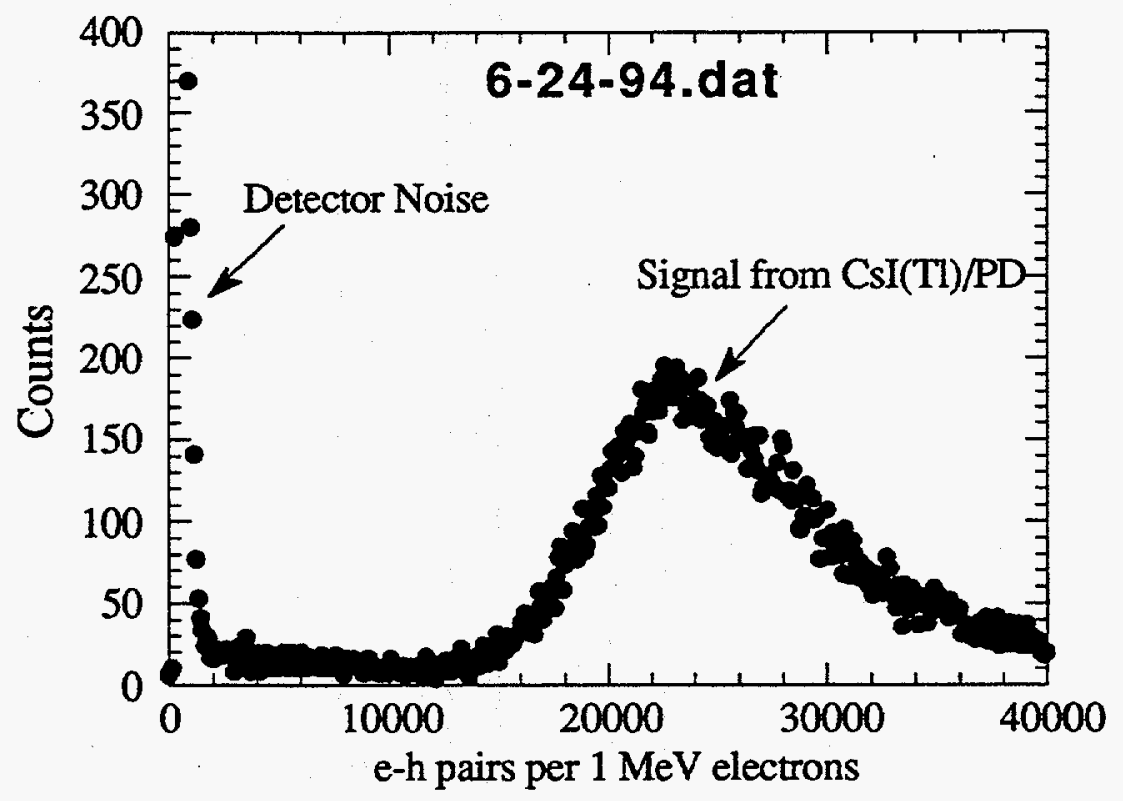

(b)

Fig. 11 MIPs measurement (a) experimental setup (b) pulse height distribution $1 \mathrm{MeV}$ betas 
(b) Pixel Array Detection of Thermal Neutrons

Thermal neutron detection with position sensitivity is useful for neutron radiography and for neutron crystallography in locating the position of hydrogen atoms in the crystal lattice. The thermal neutrons can be detected in a pixel array by use of gadolinium converters plated on to the metallic contacts of 30 $\mu \mathrm{m}$ thick a-Si:H diodes. Natural gadolinium contains various isotopes whose cross section for thermal neutron capture is 46,000 barns followed by the emission of an $80 \mathrm{KeV}$ electron internal conversion line. A $20 \mu \mathrm{m}$ thick a-Si:H diode can absorb the full energy of these conversion electrons to yield a signal of $\sim 16,000$ electron-hole pairs. Monte Carlo calculations and experimental measurements show that a $2 \mu \mathrm{m}$ thick gadolinium layer in such a combination yields a detection efficiency of $\sim 25 \%(19)$. Background from $\gamma$ rays is negligible in such a detector, due to their low interaction in the gadolinium and the a-Si:H. Further calculation shows that if we use the enriched isotope Gd-157 with a thermal neutron capture cross section of $\sim 250,000$ barns we can achieve a detection efficiency of $>63 \%$.

(c) Radionuclide Chromatography

For many biological samples, the trace radionuclides are $\mathrm{H}^{3}, \mathrm{P}^{32}$ and $\mathrm{S}^{35}$ which are $\beta$ emitting isotopes with energies of $18 \mathrm{KeV}, 1.7 \mathrm{MeV}$ and $167 \mathrm{KeV}$ respectively. A pixel array with elements $100 \times 100 \mu \mathrm{m}$ in size using either of the two schemes thick a-Si:H or thin photodiodes with a CsI(TI) scintillator layer $400 \mu \mathrm{m}$ thick can be used to map the distributions. For the $\mathrm{P}^{32}$ and $\mathrm{S}^{35}$ using the high energy portion of the spectrum, it is possible to count individual events. A a-Si:H layer can store charge for a few minutes and the integrated flux, if it is adequate above amplifier and system noise can be recorded(20) for the $H^{3}$. 
(d) Medical Imaging

At present the detectors used in medical imaging are (a) X-ray film coupled to intensifying screens Lanex and Chronex manufactured by Kodak and DuPont respectively. (b) Real time imaging is accomplished by electronic image intensifier tubes with an X-ray sensitive layer of $\mathrm{CsI}(\mathrm{Na})$ deposited internally on the cathode. Both of these have a spatial resolution-given as an MTF (Modulation Transfer Function) which has a value of 5-6 line pairs per $\mathrm{mm}$ at the $10 \%$ level. A possible solid state replacement is a pixel array of a-Si:H photodiodes with either a Lanex or $\mathrm{CsI}(\mathrm{TI})$ scintillator as the $X$-ray converter. Prototype arrays with a pitch of $127 \times 127 \mu \mathrm{m}$ have been made by Xerox PARC.(21) The MTF of those devices is $\sim 5$ line pairs per $\mathrm{mm}$ at $10 \%$ level. We have measured the MTF of our CsI(TI) columnar layers with $\mathrm{X}$-rays of 41 and $90 \mathrm{KV}$. The MTF of our $\mathrm{CsI}(\mathrm{TI})$ layers as a function of thickness is shown in Fig.12. These layers have X-ray detection sensitivities $\sim 3$ times higher than those of the comparable Lanex screens when measured at the same resolution. We are presently testing pixel diode arrays with pixel size $50 \times 50$ $\mu \mathrm{m}$ coupled to $\mathrm{Csl}(\mathrm{TI})$ layers $150 \mu \mathrm{m}$ thick to give an overall MTF of $10 \mathrm{lp} / \mathrm{mm}$.

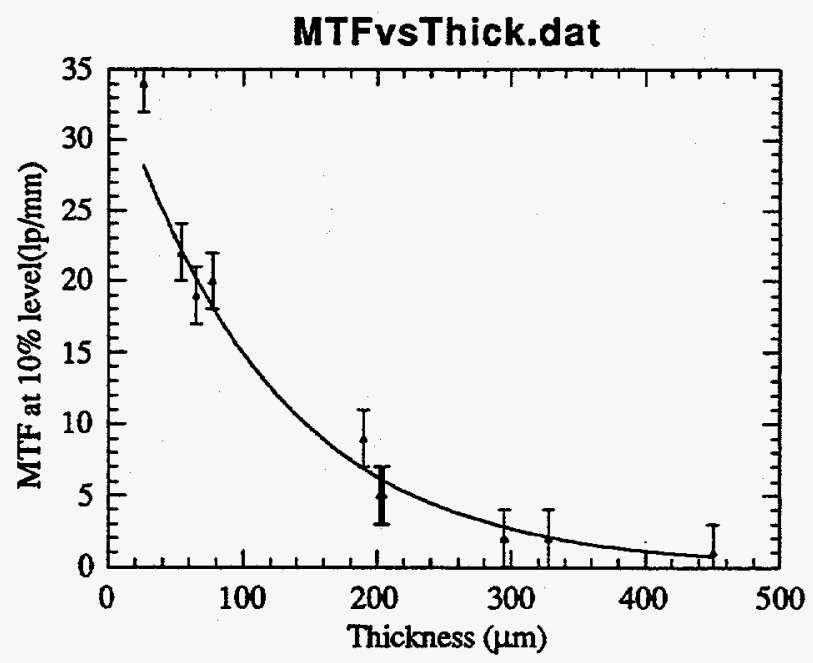

Fig. 12 Resolution: line pairs/mm as function of CsI thickness 


\section{SUMMARY AND CONCLUSIONS}

At present both the thick diode arrays and the thin photodiode/scintillator layer are capable of detecting MIPs for particle and nuclear physics. The thick a-Si:H diodes which can now be produced with sufficiently low mechanical stress have the relative advantage that their radiation resistance in high neutron and charged particle environments is higher than the CsI(TI) scintillator. Both are equally satisfactory for radionuclide chromatography. Neutron crystallography can be done with gadolinium/a-Si:H pixel arrays. $\gamma$-ray crystallography can be done with moderately thick 20 $\mu \mathrm{m}$ a-Si:H/a-Ge:H detectors.

Real time imaging of $\mathrm{X}$-ray distributions in medicine (digital radiography) is done best with the columnar CsI(TI)/a-Si:H pixel arrays. Satisfactory readout of pixel arrays can be done with a-Si:H TFT switches, or with one or two diode/pixel readout schemes. Polysilicon charge sensitive amplifiers are available for single event readout. However since they require multi layer deposition technology their implementation will occur at a later time.

\section{ACKNOWLEDGEMENTS}

We would like to thank Drs. R. A. Street and M. Hack from Xerox PARC for many useful discussion. We also would especially like to thank Dr. Gene Weckler from EG\&G Reticon for lending us many EG\&G linear detector strips which were essential for our resolution measurements and for many useful technical discussions. We also would like to thank the staff of the Materials Research Group, Wheat Ridge, Colorado for making some our thick a-Si:H diode in the past.

This work was supported by the Director, Office of Energy Research, Office of High Energy and Nuclear Physics, and Office of Health and Environmental Research, Division of Physics and Technological Research of the US. Department of Energy under contract No. DE-AC03-76SF00098 


\section{REFERENCES:}

[1] Y. Kitsuno, G.Cho, J. Drewery, W.S.Hong, V. Perez-Mendez, Jpn. J. App.

Phys.,33 (1994) 1261-1267

[2] T. Pochet, A. Ilie, F. Foulon, B. Equer, IEEE Trans. Nuc. Sci. NS41 (1994), 10141018.

[3] R. A. Street, Phys. Rev. B 27 (1983) 4294.

[4] S. Qureshi, V. Perez-Mendez, S. N. Kaplan, I. Fujieda, G. Cho, R. A. Street, Mat. Res. Symp. 149 (1989) 649-654.

[5] I. Fujieda, G. Cho, J. Drewery, S. N. Kaplan, V. Perez-Mendez, S. Qureshi, D. Wildermuth, R. A. Street, Mat. Res. Symp. 192 (1990) 399-404.

[6] T. Pochet, J. Dubeau, L. A. Hamel, B. Equer, A. Karak, Mat. Res. Soc. 149 (1989) 661. Also B. Equer Mat. Res. Soc. 258 (to be published).

[7] J. Drewery, G. Cho, T. Jing, S. Kaplan, A. Mireshghi, V. Perez-Mendez, D. Wildermuth, to be published Mat. Res. Soc. 258 (Oct. 1992).

[8] R. A. Street, J. Zesch, M. J. Thompson, App. Phys. Letters 43 (193) 672-676.

[9] G. Cho, S. Qureshi, J. Drewery, T. Jing, S. N. Kaplan, A. Mireshghi, V. PerezMendez, D. Wildermuth, IEEE Trans. Nuc. Sci. NS 39 (1992), 641-644.

[10] V. Perez-Mendez, Chapter 8 in "Physics and Applications of Amorphous and Microcrystalline Semiconductor Devices", J. Kanicki, Ed., Artech House Pub., Boston, MA (May 1991). This chapter has references to work published at that time.

[11] L. Holl, E. Lorenz, G. Mageras, IEEE Trans. Nuc. Sci NS 35 (1988) 105-109.

[12] C. W. Bates, Adv. Electronics and Electron Physics 28A (1968) 451-459.

[13] A.L.N. Stevels and A.D.M. Schrama de Pauw, Phillips Res. Rpts. 29 (1974) 340362.

[14] T. Jing, C. A. Goodman, J. Drewery, G. Cho, W.S. Hong, H. Lee, S. N. Kaplan, A. Mireshghi, V. Perez-Mendez, D. Wildermuth, IEEE Trans. Nuc. Sci NS 41 (1994) 903-909. 
[15] S. A. Kleinfelder, W. C. Carithers, R. P. Ely, C. Haber, F. Kirsten, H. Spieler, IEEE Trans. Nuc. Sci. NS-35 (1988) 171.

[16] G. Cho, J. Drewery, W. S. Hong, T.Jing, H. Lee, S. N. Kaplan, a. Mireshghi, V. Perez-Mendez, D. Wildermuth, Mat. Res. Symp. (1993) 969-974.

[17] G. Cho, V. Perez-Mendez, M. Hack and A. Lewis, LBL-32219 (April 1992). T Mat. Res. Soc 258 (Oct 1992) 1181-1186.

[18] G. Giakoumakis, App. Phys. A 52 (1991) 7-9.

[19] A. Mireshghi, G. Cho, J. Drewery, T. Jing, S. N. Kaplan, V. Perez-Mendez and D. Wildermuth, LBL-31410, IEEE Trans.Nuc. Sci., NS-39, (1992). 635-640 [20] H. Lee, G. Cho, J.Drewery, W. Hong, S.N. Kaplan, A. Mireshghi, V. Perez-Mendez, D.wildermuth, Mat. Res. Symp. 292 (1993) 1023-1028.

[21] L. Antonuk, J. Boudry, Y. El-mohri, W. Huang, J. Siewerosen, J. Yorkston, R. A. Street, SPIE, Physics of Medical Imaging, 2163(1994) 118-128 